\section{Computational Cost Reduction Using Coincident Boundary Microphones for Convolutive Blind Signal Separation}

\section{J.M. Sanchis and J. J. Rieta}

This work demonstrates that an acoustic mixture system can be configured to accomplish the instantaneous mixture model conditions. The system in achievable using coincident boundary microphones, which is a suitable disposition to uniform the propagation channel delays and to reduce the number of reflections characterizing the system impulse response. With the use of coincident microphones, convolutive BSS algorithms provide optimal results with reduced number of taps, thus, decreasing computational cost.

Introduction: To solve the blind source separation (BSS) problem of convolutive acoustic mixtures, the environment acoustic conditions both with the microphones placement for obtaining the observations plays an important role [1-3]. Several works have been focused on how the mixing system affects the BSS algorithms and their performance. In this sense, the acoustic environment effect has been analyzed [4], evidencing that a large impulse response modeling the mixing system, which is directly related with the reverberation time and the density of reflections characterizing the acoustic response of the chamber, gives poor separation results.

However, the transducers characteristics and placement employed for recording the observations (being, at the same time, the inputs of the BSS algorithm), have been evaluated in a few number of studies $[5,6]$. Until now, the available bibliography regarding convolutive BSS always establishes the separated microphones technique, usually at equispaced points, as the placement procedure for the microphone array [5]. In contrast to this technique, the use of coincident boundary microphones together with an adequate transducer placement, able to minimize the number of reflections captured by the microphones, is presented in this work.

To analyze the proposed mixing system structure for two sources and two observations (see Fig. 1 for details), it is possible to define $\mathbf{s}[n]=\left[s_{1}[n] s_{2}[n]\right]^{T}$ as the sources vector, $\mathbf{x}[n]=\left[x_{1}[n] x_{2}[n]\right]^{T}$ as the observations vector and $h_{j i}[n]$ as the impulse response of the LTI system connecting the $i^{t h}$ observation with the $j^{\text {th }}$ source $(i, j=1,2)$. Therefore, we can express the mixture model as

$$
\left[\begin{array}{l}
x_{1}[n] \\
x_{2}[n]
\end{array}\right]=\left[\begin{array}{ll}
h_{11}[n] & h_{12}[n] \\
h_{21}[n] & h_{22}[n]
\end{array}\right] *\left[\begin{array}{l}
s_{1}[n] \\
s_{2}[n]
\end{array}\right]
$$

Using the coincident microphones methodology (see Fig. 1.b), instead of the traditional separated procedure (Fig. 1.a), the acoustic signal will ideally reach both microphones at the same time instant but, due to the directional behaviour of the microphones, each source will be captured with different power level. Consequently, two observations or differently mixed signals are obtained in this manner but, on the other hand, there are no time differences between the sources present at each mixture. Hence, it is possible to obtain an instantaneous mixing system for real world acoustic signals. Moreover, with the use of coincident surface microphones it will be possible to reduce the number of received reflections, thus simplifying the impulse response of the mixing system.

Considering that the directive property of the $i^{\text {th }}$ microphone towards the $j^{t h}$ source, $d_{j i}$, is frequency independent, the path impulse response can be decomposed into a directivity factor, $d_{j i}$, and a term reflecting the acoustic environment characteristics, $h_{j i}^{\prime}[n]$

$$
h_{j i}[n]=d_{j i}[n] h_{j i}^{\prime}[n]
$$

in addition, when two microphones are summoned at the same point, the impulse response from a certain source towards each of the microphones can be supposed to be approximately the same, hence

$$
h_{11}^{\prime}[n]=h_{21}^{\prime}[n]=h_{1}^{\prime}[n] \quad ; \quad h_{12}^{\prime}[n]=h_{22}^{\prime}[n]=h_{2}^{\prime}[n]
$$

with these considerations, we can rewrite Eq. 1 as

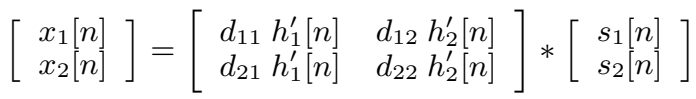

and concentrating the acoustic environment impulse response

$$
\left[\begin{array}{l}
x_{1}[n] \\
x_{2}[n]
\end{array}\right]=\left[\begin{array}{ll}
d_{11} & d_{12} \\
d_{21} & d_{22}
\end{array}\right] \cdot\left[\begin{array}{l}
h_{1}^{\prime}[n] * s_{1}[n] \\
h_{1}^{\prime}[n] * s_{2}[n]
\end{array}\right]
$$

Therefore, Eq. 5 shows that the observations captured by the microphones can be considered as an instantaneous mixture of the source

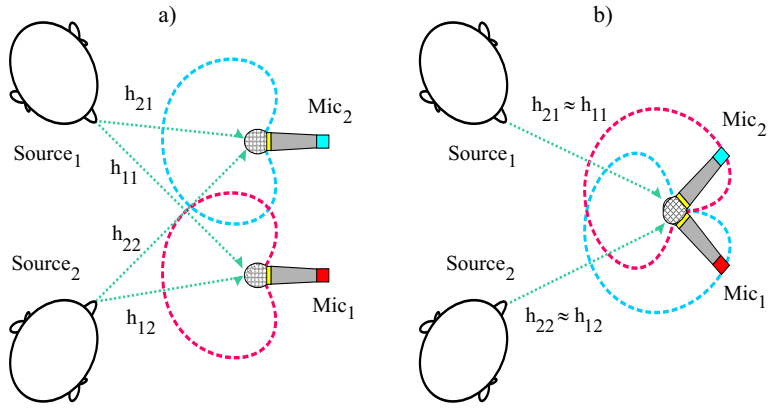

Figure 1: a) Recording scheme using traditional separated microphones. b) Proposed coincident microphones disposition.
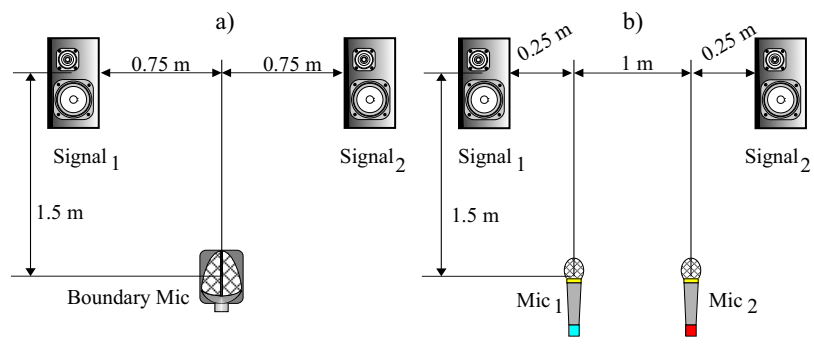

Figure 2: Transducers and sensors configuration inside the acoustic chamber used to evaluate the microphones influence according to coincident (a) and separated schemes (b).

signals modified by the effect of a concrete acoustic environment. As a consequence, the situation can be transformed into an instantaneous mixture separation problem. If this problem were ideally solved, it would be possible to separate the sources perfectly, each one being affected by the acoustic environment of the hall. Therefore, in those situations where the main objective is the spatial separation of the acoustic sources and the minimisation of the interferences introduced by other sources, this perspective of the problem would be optimal. However, in practical situations it could be more complicated to accomplish the requirements established in this model. Firstly, a small positioning error will exist in the transducers conforming the coincident array and, secondly, the microphone directivity pattern does not remain constant with frequency.

Experiments: To corroborate the previous ideas, experiments using both microphones configuration, coincident and separated, have been carried out. These experiments have consisted of a simple scenario of two sources and two sensors $(2 \times 2)$ summoned into two different acoustic environments: an anechoic chamber and a recording studio. These configurations are illustrated in Fig. 2.

The recorded observations were computed using the selected BSS algorithm and the influence of the filter tap number in the separation performance was analyzed, ranging from 1 (instantaneous mixture) up to the maximum impulse response length associated to each situation. In this work, the tested BSS algorithms have been the CoBliSS [7] and MBLMS algorithms [8].

To validate empirically the proposed methodology, the autocorrelation and the cross-correlation sequences between the obtained impulse responses $h_{11} / h_{21}$ and $h_{12} / h_{22}$ have been computed using the coincident microphones configuration. As an example, Fig. 3 plots the cross-correlation and autocorrelation functions of the impulse responses $h_{11}$ and $h_{21}$ in an anechoic chamber for coincident microphones.

Results: The independent sources used in the experiments have been estimated by applying the convolutive BSS algorithms [7,8] and, making use of the filter tap number variation, the separation performance has been measured according to the signal to interference ratio (SIR) defined in [9]. Results obtained with coincident and separated microphones are compared in Fig. 4 for the anechoic chamber and the recording studio. We can observe that: firstly, the convolutive BSS algorithm obtained better results in both acoustic environments for short filter lengths. Moreover, the highest SIR is reached with the minimum number of taps, i.e., for an instantaneous linear mixing system. Secondly, as can be seen in the SIR plots, the results obtained with coincident microphones seem to be independent of the acoustic characteristics of the enclosure. 


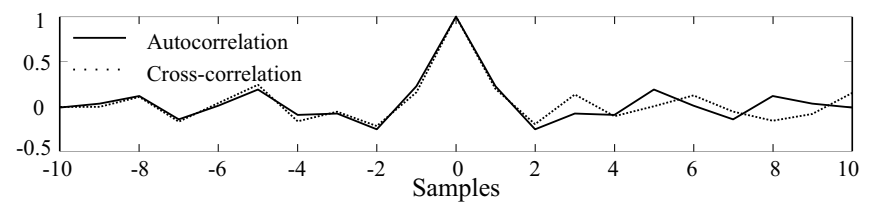

Figure 3: Cross-correlation and autocorrelation functions of the impulse responses $h_{11}$ and $h_{21}$ in an anechoic chamber for coincident microphones $\left(f_{s}=44100 \mathrm{~Hz}\right)$.

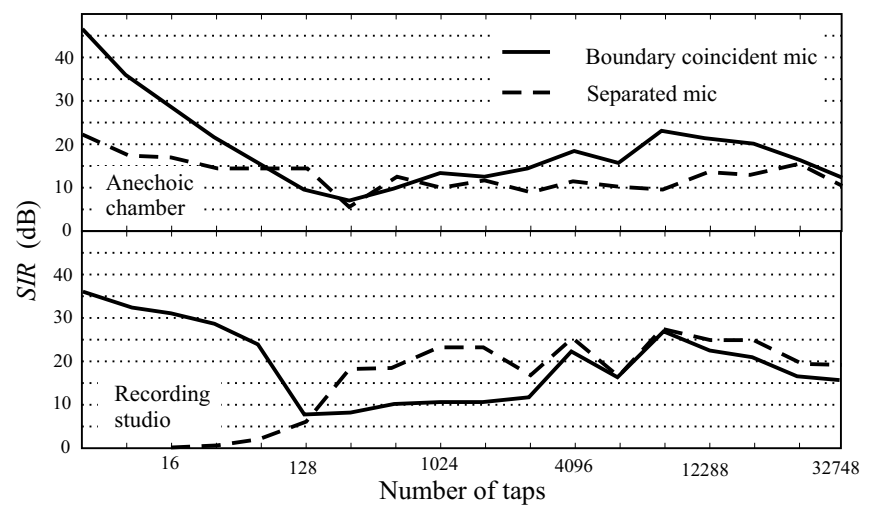

Figure 4: SIR for the same BSS algorithm [7,8] in anechoic chamber and recording studio for coincident and separated microphones.

Conclusion: The present work has demonstrated that optimal convolutive BSS results are achievable, with the use of coincident boundary microphones, reducing considerably the filter taps number in simple configurations of two sources and two sensors. In addition, a substantial decrease in the computational cost can be reached by using the instantaneous approximation, where it is possible to recover the source signals convolved with the impulse response of the chamber but, at the same time, with the highest SIR.

(c) IEE

4th October 2004

Electronic Letters Online No: 2004XXXX

Juan Manuel Sanchis, José Joaquín Rieta (Electronic Engineering Dep., Valencia University of Technology, Carretera Nazaret-Oliva s/ $n, 46730$, Gandía, Valencia, Spain)

E-mail: jmsanch@eln.upv.es

\section{References}

[1] H. Sahlin and H. Broman, "Signal separation applied to real world signals," International Workshop on Acoustic Echo and Noise Control, pp. 73-76, Imperial College London, London, UK, September 1997.

[2] D. Schobben, K. Torkkola, and P. Smaragdis, "Evaluation of blind signal separation methods," International Conference on Independent Component Analysis and Blind Signal Separation (ICA), vol. 1, pp. 261-266, Aussois, France, January 1999.

[3] J. M. Sanchis, Evaluation of mixture conditions in convolutive blind source separation for audio applications. Ph.D. Thesis, Valencia University of Technology (Spain), December 2003.

[4] R. Mukai, S. Araki, and S. Makino, "Separation and dereverberation performance of frequency domain blind source separation," International Conference on Independent Component Analysis and Blind Signal Separation (ICA), vol. 4, pp. 230-235, San Diego, California, USA, December 2001.

[5] D. Rabinkin, R. Renomeron, J. French, and J. Flanagan "Optimun microphone placement for array sound capture," Meeting of the Acoustical Society of America, vol. 3162, pp. 227-239, State College, Pennsylvania, USA, June 1997.

[6] J. Hopgood, P. Rayner, and P. Yuen, "The effect of sensor placement in blind source separation," IEEE Workshop on Applications of Signal Processing to Audio and Acoustics, vol. 1, pp. New Paltz, New York, USA, October 2001.

[7] D. Schobden, Real-time adaptative concepts in acoustics. Kluwer Academic Publishers, 2001.

[8] R. Lambert, Multichannel Blind Deconvolution: FIR Matrix Algebra and Separation of Multipath Mixtures. Ph.D. Thesis, University of Southern California, (USA), 1996

[9] S. Araki, R. Mukai, S. Makino, T. Nishikawa, and H. Saruwatari, "The fundamental limitation of frequency domain blind source separation for convolutive mixture of speech," IEEE Transactions on Speech and Audio Processing, vol. 11, no. 2, pp. 109-116, March 2003. 